\title{
A New Active Locomotion Capsule Endoscopy under Magnetic Control and Automated Reading Program
}

\author{
Dong Jun Oh${ }^{1}$, Kwang Seop Kim² and Yun Jeong Lim ${ }^{1}$ \\ ${ }^{1}$ Department of Internal Medicine, Dongguk University Ilsan Hospital, Dongguk University College of Medicine, Goyang, ${ }^{2}$ Chief \\ Research Engineer, Research and Development team, IntroMedic Co., Ltd., Seoul, Korea
}

Capsule endoscopy (CE) is the first-line diagnostic modality for detecting small bowel lesions. CE is non-invasive and does not require sedation, but its movements cannot be controlled, it requires a long time for interpretation, and it has lower image quality compared to wired endoscopy. With the rapid advancement of technology, several methods to solve these problems have been developed. This article describes the ongoing developments regarding external CE locomotion using magnetic force, artificial intelligence-based interpretation, and image-enhancing technologies with the CE system. Clin Endosc 2020;53:395-401

Key Words: 3-D imaging; Artificial intelligence; Capsule endoscopy; Locomotion; Magnetics

\section{INTRODUCTION}

In 2001, the Food and Drug Administration (FDA) approved the application of small bowel (SB) capsule endoscopy (CE), ${ }^{1}$ which was introduced to Korea the following year. ${ }^{2} \mathrm{CE}$ is a very useful diagnostic modality for detecting SB diseases including obscure gastrointestinal (GI) bleeding, Crohn's disease, SB tumors, and polyposis syndrome. ${ }^{3,4}$ An advantage of $\mathrm{CE}$ is that it is a non-invasive, non-sedative procedure and allows direct mucosal visualization. ${ }^{5}$ However, gastric retention or delayed transition of CE may lead to incomplete SB examination and may not detect SB lesions. ${ }^{6}$ Also, the interpretation of CE images usually takes more than an hour, which can be a tedious process for clinicians. ${ }^{7}$ In addition, enhancement of the CE image quality is needed to improve the diagnostic

Received: May 8, 2020 Accepted: May 28, 2020

Correspondence: Yun Jeong Lim

Department of Internal Medicine, Dongguk University Ilsan Hospital, Dongguk University College of Medicine, 27 Dongguk-ro, Ilsandong-gu, Goyang 10326, Korea

Tel: +82-31-961-7133, Fax: +82-31-961-7730, E-mail: drlimyj@gmail.com ORCID: https://orcid.org/0000-0002-3279-332X

(c) This is an Open Access article distributed under the terms of the Creative Commons Attribution Non-Commercial License (http://creativecommons.org/ licenses/by-nc/3.0) which permits unrestricted non-commercial use, distribution, and reproduction in any medium, provided the original work is properly cited. accuracy. To resolve these problems, many studies have been conducted on active locomotion, artificial intelligence (AI)based interpretation, and image enhancement of CE. This article reviews the newly developed diagnostic and interpretation technologies of CE.

\section{ACTIVE LOCOMOTION UNDER MAGNETIC CONTROL}

CE is moved by gut peristalsis and gravity. ${ }^{6,8}$ So, there is a possibility of incomplete $\mathrm{SB}$ examination if the $\mathrm{CE}$ remains stagnant in a specific area of the stomach or SB. ${ }^{9}$ Although a longer battery life improved the success rate of complete $\mathrm{SB}$ examination, ${ }^{10}$ incomplete SB examination has still not been solved. Moreover, there is a need for non-invasive gastric examinations with the advantages of $\mathrm{CE}$, but gastric examination using CE is impossible due to the wide lumen of the stomach. ${ }^{11}$ Therefore, many studies regarding active locomotion in $\mathrm{CE}$ have been conducted for complete gastric examinations and reductions of the pyloric transit time.

The active locomotion systems of CE are generally divided into internal and external locomotion. ${ }^{12}$ Some studies have been conducted on the internal locomotion that controls CE movements by using paddling, legs, and propellers within the 
GI tract. ${ }^{12}$ However, internal locomotion was problematic due to high power consumption and unstable movements, and it was impossible to integrate the advanced technology for internal locomotion in a small capsule. ${ }^{13}$ Therefore, external locomotion using magnetic force has emerged as a feasible solution. ${ }^{14}$ In 2010, human gastric examination with CE using magnetic manipulation was first published. ${ }^{15}$ Since then, another study validated the use of an external magnetic controller for gastric examinations with $\mathrm{CE} .{ }^{16}$ An external magnetic controller manipulates CE movements through rotations, tilting, and jumping. ${ }^{17}$ It significantly reduced the median pyloric transit time in the gastric examination (4.7 minutes vs. 58.4 minutes in study and control groups, respectively). ${ }^{18}$ Currently, magnetic capsule endoscopy (MCE) is the main trend of external locomotion, and complete stomach and SB examination is becoming possible. There are currently several leading companies that have developed MCE with active locomotion for gastric examinations. ${ }^{11}$

\section{Siemens Healthcare and Olympus Medical Systems Co.}

Siemens Healthcare (Erlangen, Germany) and Olympus Medical Systems Co. (Center Valley, PA, USA and Tokyo, Japan) jointly developed an MCE system applying an electromagnetic coil that manipulates a joystick controller to navigate the stomach. In a study that utilized the MCE system for the gastric examinations of 53 participants, the feasibility of the MCE system was confirmed as the antrum, body, fundus, and cardia were fully visualized in $98 \%, 96 \%, 73 \%$, and $75 \%$ of cases, respectively. ${ }^{19}$ Moreover, another study was conducted to compare the effectiveness of this MCE system and esophagogastroduodenoscopy (EGD) in identifying gastric lesions, such as inflammation, polyps, and ulcers. Among the 61 patients, the MCE system missed 14 lesions, but EGD missed 31 lesions. The overall diagnostic accuracy was similar between the two methods, indicating the potential for MCE as a screening method in high incidences of gastric cancer. ${ }^{17}$ However, it is not currently commercially available. ${ }^{20}$

\section{IntroMedic Co., Ltd.}

Mirocam-Navi system, developed by IntroMedic Co., Ltd. (Seoul, Korea), applies novel technology that uses the human body as a conductive medium to transmit data from CE to the electrodes attached to the body. ${ }^{21}$ IntroMedic Co., Ltd. developed a hand-held magnetic controller as a new navigation system. When a gastric examination was performed using the Mirocam with a hand-held magnetic controller (Mirocam-Navi system), the landmarks of the stomach were successfully visualized (88\%-100\%). Thus, the Mirocam-Navi system was verified to be feasible for gastric examinations. ${ }^{22}$ In addition, a hand-held magnetic controller has fewer space restrictions compared to the large-sized computer navigation systems of other companies. Moreover, well-experienced endoscopists using the Mirocam-Navi system may be able to inspect the lesions more accurately and shorten the examination time. ${ }^{23}$ The Mirocam-Navi system had satisfactory maneuverability, mucosal visualization, and patient tolerance in esophageal, duodenal, and stomach examinations. ${ }^{24}$ When the Mirocam-Navi system and EGD were compared among 33 patients with suspected acute GI bleeding, the diagnosis accuracy of focal lesions was higher in the Mirocam-Navi system (40 vs. $25, p=0.02$ ), and bleeding focus in the SB was more likely detected by the Mirocam-Navi system than by EGD (18\%). ${ }^{25}$ In addition, when the efficacy of the Mirocam-Navi system and EGD alone were compared among 49 patients with recurrent or iron-refractory iron deficiency anemia, the Mirocam-Navi system, which can combine examinations of the upper GI and entire SB, was more likely to detect pathologic lesions than EGD alone (113 vs. $52, p<0.001) .{ }^{26}$ Overall, the Mirocam-Navi system has high diagnostic accuracy and patient-friendly comfort for upper GI examinations.

\section{Ankon Technology Co., Ltd.}

At first, manual magnetic controllers showed good performances, but in the initial stages, the magnetic force was insufficient for pyloric passage ${ }^{16}$ and an animal study showed that robotic magnetic control was more accurate than manual control. ${ }^{27}$ Therefore, Ankon Technology Co., Ltd. (Shanghai, China) developed a C-arm-shaped robotic magnet controller and examined the stomach of healthy volunteers. In this study, the good maneuverability, detectability, and safety of the robotic MCE system was confirmed. ${ }^{28}$ In several studies conducted using the robotic MCE system, the robotic MCE system showed high diagnostic accuracy similar to EGD, but less discomfort than EGD. ${ }^{29,30}$ Moreover, it demonstrated a high sensitivity in detecting superficial gastric neoplasias. ${ }^{31}$ Based on the valuable outcomes of these studies, a large-scale gastric cancer screening study involving 3,182 asymptomatic patients was undertaken using the robotic MCE system. In this study, gastric cancer was diagnosed in 7 people $(0.22 \%)$. Since this rate was similar in Korea and Japan (approximately $0.2 \%$ ), the robotic MCE system can be used as a screening tool for gastric cancer. ${ }^{32}$ Because the MCE system shows a good diagnostic detection rate for gastric lesions, it can be a good alternative for EGD in high-risk patients. ${ }^{33}$ Furthermore, pediatric patients, who are unable to tolerate EGD, can safely undergo successful gastric examination with the robotic MCE system. ${ }^{34}$ Owing to its high diagnostic performance and safety, the robotic MCE system for human gastric examinations is currently approved by the Chinese FDA. ${ }^{35}$ Recently, a second-generation MCE has 
been developed, which has greater image resolution, shortened examination duration, and improved maneuverability compared to the first-generation MCE. ${ }^{36}$

\section{ARTIFICIAL INTELLIGENCE-BASED INTERPRETATION PROGRAM}

The interpretation of numerous SB images acquired by $\mathrm{CE}$ requires long processing times and high concentration from clinicians. ${ }^{37}$ To solve this problem, a study was conducted to interpret $\mathrm{CE}$ images using a computer-aided diagnostic tool. This tool analyzed the images using characteristic colors of the lesions; however, it was difficult to use in real clinical practice because of its low sensitivity and specificity. ${ }^{38}$ In a recent guideline, pre-interpretation by qualified nurses and trained technicians is recommended to reduce the burden and interpretation time on clinicians. ${ }^{39}$ However, automated interpretation of $\mathrm{CE}$ has gained much attention with the development of AI technology, which had been firstly used in $1955 .{ }^{40}$ In the history of AI technology, the era of deep learning began in $2010 .{ }^{41}$ In 2012, the most well-known image recognition competition, ImageNet Classification, emerged. The first deep-learning model based on the convolutional neural network (CNN) model was showcased, and it significantly reduced the error rate compared to previous models. ${ }^{42}$ The $\mathrm{CNN}$ model has presented outstanding outcomes in the field of medical image analysis and become the most preferred deep learning method in the field of medicine. ${ }^{43}$ Several studies proved that the $\mathrm{CNN}$ model was superior to conventional machine learning models in terms of lesion analysis, ${ }^{44}$ so studies that utilized $\mathrm{CNN}$-based interpretations for detecting SB lesions have been conducted since 2010. When CNN-based interpretations were used for the detection of angioectasia, the most common $\mathrm{SB}$ vascular lesion, it demonstrated excellent sensitivity and specificity close to $100 \%{ }^{45,46}$ In addition, CNN-based inter- pretations showed an accuracy of $90.8 \%$ in the diagnosis of SB erosions and ulcers ${ }^{47}$ and showed a higher diagnostic accuracy in blood contents (sensitivity, 96.63\%; specificity, 99.96\%) compared to conventional suspected blood indicators. ${ }^{48}$ In a large-scale study in China, a new CNN interpretation model based on 113,426,569 images was developed, which showed a higher sensitivity (77.9\%-99.9\%) and lesion detection rate (54.6\%-70.9\%) compared to conventional CE interpretations for detecting various SB lesions, such as inflammation, ulcers, and polyps. The reading time was markedly shortened from 99.6 minutes to 5.9 minutes. ${ }^{49}$ Important studies on CE interpretations using the CNN model are summarized in Table 1.

\section{IMAGE-ENHANCING TECHNOLOGIES}

It is important to improve the quality of images to increase the diagnostic accuracy of $\mathrm{CE}$. Currently, the imaging enhancing methods of $\mathrm{CE}$ include 3D image reconstruction, capsule chromo-endomicroscopy, and improvements of the image resolution using de-noising and de-blurring processes.

A study showed that $3 \mathrm{D}$ reconstruction of the $\mathrm{CE}$ image may be helpful for inexperienced CE readers since elevated lesions observed in CE may be subepithelial lesions or normal variants. ${ }^{50}$ IntroMedic Co., Ltd. recently commercialized a 3D CE equipped with a dual-stereo camera (MiroCam ${ }^{\circledR}$ MC 4000) which allows for 3D reconstruction (Supplementary video 1) and size measurements (Fig. 1). The entire SB examination was performed using this $3 \mathrm{D} \mathrm{CE}$, and its safety and feasibility were examined. ${ }^{51}$

Unlike wired endoscopy, CE cannot be used to perform biopsies for histopathologic diagnosis. ${ }^{6}$ Thus, for optical biopsies, tethered capsule endomicroscopy (CE equipped with optics capable of obtaining cross-sectional images of the gut layer) was used. ${ }^{52}$ In this study, 6 healthy patients and 7 patients with Barrett's esophagus swallowed the tethered capsule,

Table 1. Sensitivity and Specificity of the Detection of Small Bowel Lesions in Clinical Trials Using the Convolutional Neural Network-Based Interpretation

\begin{tabular}{lccc}
\hline Study & Lesion & Sensitivity (\%) & Specificity (\%) \\
\hline Leenhardt et al. $(2019)^{45}$ & Angioectasia & 100 & 96 \\
Ding et al. $(2019)^{49}$ & Vascular disease & 98.9 & 100 \\
Tsuboi et al. $(2020)^{46}$ & Angioectasia & 98.8 & 98.4 \\
Aoki et al. $(2020)^{48}$ & Blood contents & 96.6 & 99.9 \\
Ding et al. $(2019)^{49}$ & Bleeding & 100 & 100 \\
Aoki et al. $(2019)^{47}$ & Ulcers, erosion & 88.2 & 90.9 \\
Ding et al. $(2019)^{49}$ & Ulcer & 99.7 & 100 \\
\hline
\end{tabular}




\section{Ce cuncan enooscopy}
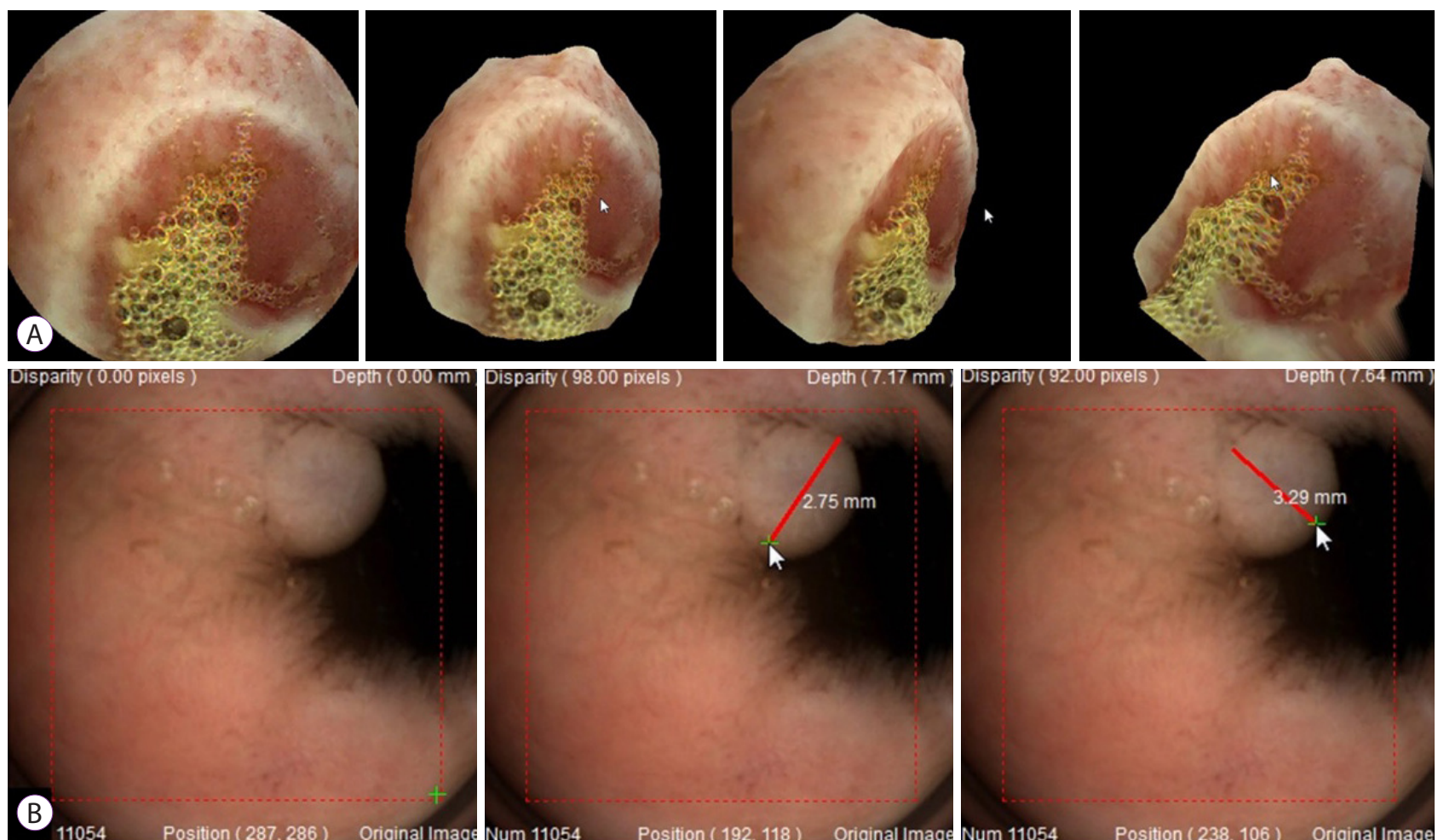

Depth $(7.64 \mathrm{~mm}$

Fig. 1. Image-enhancing technology for accurate identification of the lesions. The 3D reconstruction (A) and size measurements (B) by a Mirocam viewer (Miroview ${ }^{\circledR}$ MC 4000; IntroMedic Co., Ltd., Seoul, Korea).
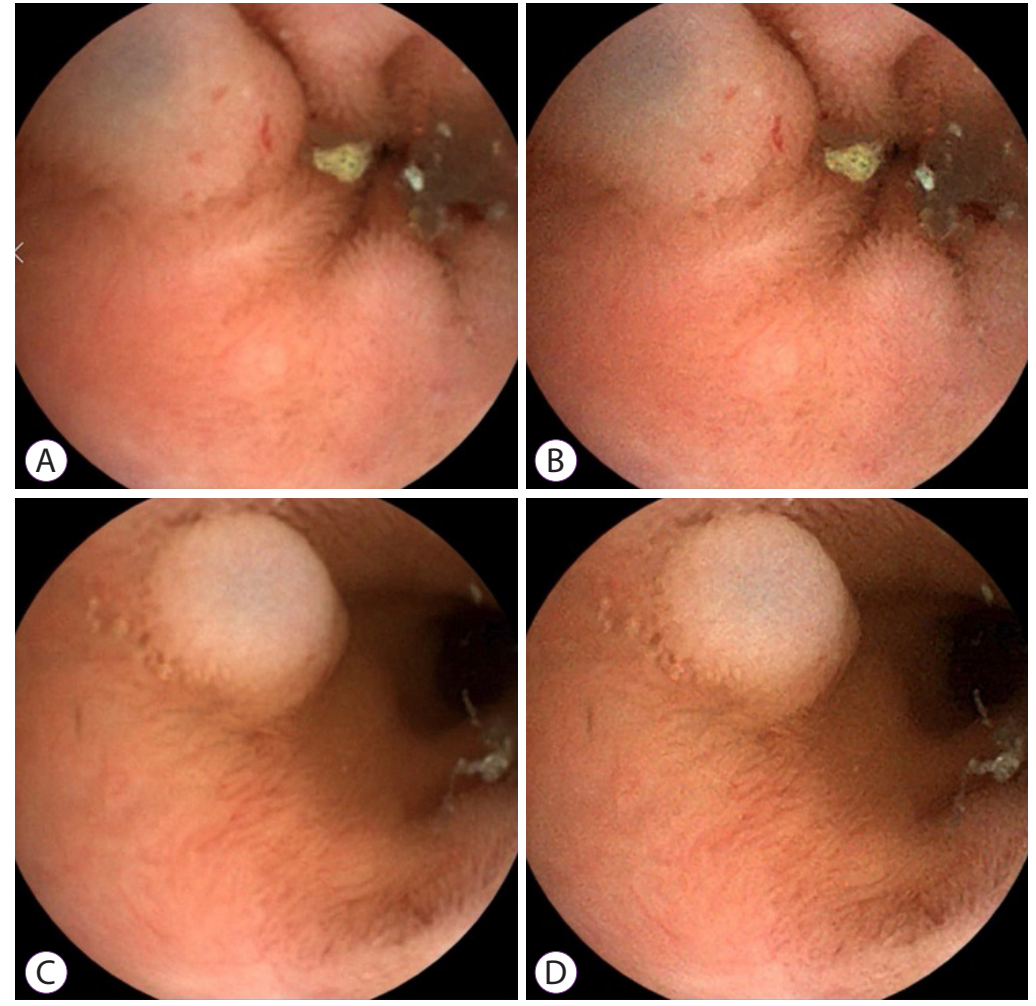

Fig. 2. Super-resolution by a Mirocam viewer (Miroview ${ }^{\circledR}$ MC 4000; IntroMedic Co., Ltd., Seoul, Korea). Erosion (A) and polyp (C) were noticed by a Miroview ${ }^{\circledR}$ MC 4000. A de-noising process, depth-guided deburring process, and deep-learning algorithm were used for super-resolution. Eventually, the clarity of the superresolution images $(B$ and $D)$ increased over the original images ( $A$ and $C)$. 


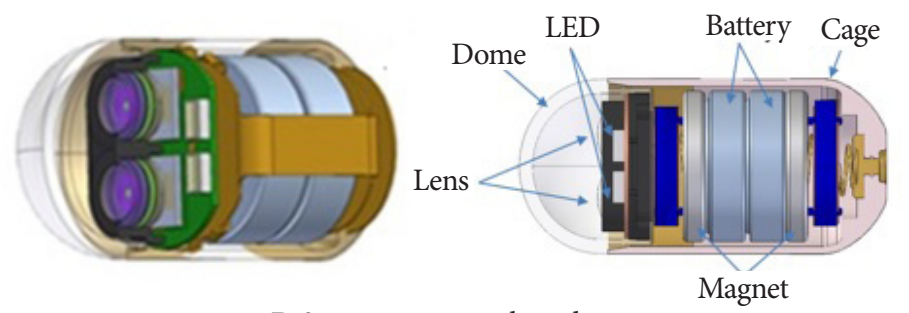

3D \& magnetic capsule endoscope

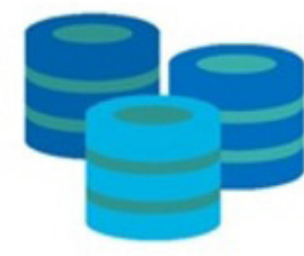

Cloud-based clinical database platform

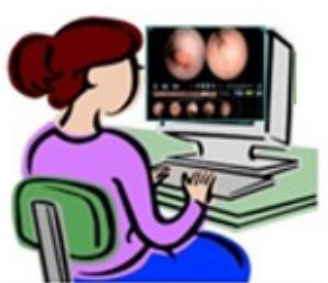

AI-based interpretation

Fig. 3. Current and future technologies of capsule endoscopy. Ultra-compact 3D wireless endoscope with active locomotion under magnetic control and artificial intelligence (Al)-based interpretation programs.

and the capsule was slowly pulled back from the stomach to the mouth for esophageal examination. The obtained 3D microstructural images accurately distinguished the normal mucosa and Barrett's esophagus. ${ }^{52}$ Capsule chromoendoscopy using flexible spectral imaging color enhancement improved the delineation of SB mucosal lesions compared with CE using conventional white light. ${ }^{53}$

The most widely used CEs in the world, Pillcam SB3 (Medtronic Co., Ltd., Minneapolis, MN, USA) and Mirocam (IntroMedic), have a resolution of $320 \times 320$ pixels, while first-generation and second-generation MCE (Ankon Technology) have a resolution of $480 \times 480$ pixels and $720 \times 720$ pixels, respectively. ${ }^{31,35,54,55}$ However, these CEs still have lower image resolutions than standard or high-definition wired endoscopies (400,000-1,000,000 pixels). ${ }^{56} \mathrm{CE}$ cannot achieve a higher resolution due to the limitations of its wireless design and small size. Studies using de-noising and de-blurring algorithms are in development to obtain clearer images (Fig. 2).

\section{CONCLUSIONS}

Magnetically controlled external locomotion and AI-based interpretation (especially $\mathrm{CNN}$ ) allow for more efficient examinations of the GI tract. In addition, 3D reconstruction, high image resolution, and capsule chromo-endomicroscopy can elucidate the endoscopic and microscopic features of the lesions, thereby reducing unnecessary invasive procedures. If the MCE system with enhanced 3D imaging and AI-based interpretation are developed, whole gut (from the mouth to the anus) screening by a single capsule, pan-endoscopy will be possible in the near future (Fig. 3).

\section{Conflicts of Interest}

Kwang Seop Kim is working as a chief research engineer in the research and development team, IntroMedic Co., Ltd., Seoul, Korea. The other authors have no financial conflicts of interest.

\section{Acknowledgements}

This study was supported by a grant (HI19C0665) from the Korean Health Technology R\&D project through the Korean Health Industry Development Institute (KHIDI), Korea

\section{Author Contributions}

Conceptualization: Yun Jeong Lim

Resources: Kwang Seop Kim

Writing-original draft: Dong Jun Oh

Writing-review\&editing: YJL

ORCID

Dong Jun Oh: https://orcid.org/0000-0002-3876-2153

Kwang Seop Kim: https://orcid.org/0000-0003-3944-2271

Supplementary Material

Video 1 . The process of interpreting lesions by rotating the $3 \mathrm{D}$ reconstructed image (https://doi.org/10.5946/ce.2020.127.v001). 


\section{REFERENCES}

1. ASGE Technology Committee, Wang A, Banerjee S, et al. Wireless capsule endoscopy. Gastrointest Endosc 2013;78:805-815.

2. Lim YJ, Lee OY, Jeen YT, et al. Indications for detection, completion, and retention rates of small bowel capsule endoscopy based on the 10year data from the Korean capsule endoscopy registry. Clin Endosc 2015;48:399-404.

3. Pennazio M, Spada C, Eliakim R, et al. Small-bowel capsule endoscopy and device-assisted enteroscopy for diagnosis and treatment of small-bowel disorders: European Society of Gastrointestinal Endoscopy (ESGE) clinical guideline. Endoscopy 2015;47:352-376.

4. Enns RA, Hookey L, Armstrong D, et al. Clinical practice guidelines for the use of video capsule endoscopy. Gastroenterology 2017;152:497-514.

5. Rondonotti E, Villa F, Mulder CJ, Jacobs MA, de Franchis R. Small bowel capsule endoscopy in 2007: indications, risks and limitations. World J Gastroenterol 2007;13:6140-6149.

6. Kwack WG, Lim YJ. Current status and research into overcoming limitations of capsule endoscopy. Clin Endosc 2016;49:8-15.

7. Kim SH, Yang DH, Kim JS. Current status of interpretation of small bowel capsule endoscopy. Clin Endosc 2018;51:329-333.

8. Iddan G, Meron G, Glukhovsky A, Swain P. Wireless capsule endoscopy. Nature 2000;405:417.

9. Höög CM, Bark L, Arkani J, Gorsetman J, Broström O, Sjöqvist U. Capsule retentions and incomplete capsule endoscopy examinations: an analysis of 2300 examinations. Gastroenterol Res Pract 2012;2012:518718.

10. Ou G, Shahidi N, Galorport C, Takach O, Lee T, Enns R. Effect of longer battery life on small bowel capsule endoscopy. World J Gastroenterol 2015;21:2677-2682.

11. Shamsudhin N, Zverev VI, Keller H, et al. Magnetically guided capsule endoscopy. Med Phys 2017;44:e91-e111.

12. Nam SJ, Lee HS, Lim YJ. Evaluation of gastric disease with capsule endoscopy. Clin Endosc 2018;51:323-328.

13. Liu L, Towfighian S, Hila A. A review of locomotion systems for capsule endoscopy. IEEE Rev Biomed Eng 2015;8:138-151.

14. Swain P. The future of wireless capsule endoscopy. World J Gastroenterol 2008; 14:4142-4145.

15. Swain P, Toor A, Volke F, et al. Remote magnetic manipulation of a wireless capsule endoscope in the esophagus and stomach of humans (with videos). Gastrointest Endosc 2010;71:1290-1293.

16. Keller J, Fibbe C, Volke F, et al. Inspection of the human stomach using remote-controlled capsule endoscopy: a feasibility study in healthy volunteers (with videos). Gastrointest Endosc 2011;73:22-28.

17. Rey JF, Ogata H, Hosoe N, et al. Blinded nonrandomized comparative study of gastric examination with a magnetically guided capsule endoscope and standard videoendoscope. Gastrointest Endosc 2012;75:373381

18. Jiang X, Qian YY, Liu X, et al. Impact of magnetic steering on gastric transit time of a capsule endoscopy (with video). Gastrointest Endosc 2018;88:746-754.

19. Rey JF, Ogata H, Hosoe N, et al. Feasibility of stomach exploration with a guided capsule endoscope. Endoscopy 2010;42:541-545.

20. ASGE Technology Committee. Magnets in the GI tract. Gastrointest Endosc 2013;78:561-567.

21. Bang S, Park JY, Jeong S, et al. First clinical trial of the "MiRo" capsule endoscope by using a novel transmission technology: electric-field propagation. Gastrointest Endosc 2009;69:253-259.

22. Rahman I, Pioche M, Shim CS, et al. Magnetic-assisted capsule endoscopy in the upper GI tract by using a novel navigation system (with video). Gastrointest Endosc 2016;83:889-895.el.

23. Lien GS, Liu CW, Jiang JA, Chuang CL, Teng MT. Magnetic control system targeted for capsule endoscopic operations in the stomach--design, fabrication, and in vitro and ex vivo evaluations. IEEE Trans Biomed
Eng 2012;59:2068-2079.

24. Lien GS, Wu MS, Chen CN, Liu CW, Suk FM. Feasibility and safety of a novel magnetic-assisted capsule endoscope system in a preliminary examination for upper gastrointestinal tract. Surg Endosc 2018;32:19371944.

25. Ching HL, Hale MF, Sidhu R, Beg S, Ragunath K, McAlindon ME. Magnetically assisted capsule endoscopy in suspected acute upper GI bleeding versus esophagogastroduodenoscopy in detecting focal lesions. Gastrointest Endosc 2019;90:430-439.

26. Ching HL, Hale MF, Kurien M, et al. Diagnostic yield of magnetically assisted capsule endoscopy versus gastroscopy in recurrent and refractory iron deficiency anemia. Endoscopy 2019;51:409-418.

27. Ciuti G, Donlin R, Valdastri P, et al. Robotic versus manual control in magnetic steering of an endoscopic capsule. Endoscopy 2010;42:148152

28. Liao Z, Duan XD, Xin L, et al. Feasibility and safety of magnetic-controlled capsule endoscopy system in examination of human stomach: a pilot study in healthy volunteers. J Interv Gastroenterol 2012;2:155-160.

29. Zou WB, Hou XH, Xin L, et al. Magnetic-controlled capsule endoscopy vs. gastroscopy for gastric diseases: a two-center self-controlled comparative trial. Endoscopy 2015;47:525-528.

30. Liao Z, Hou X, Lin-Hu EQ, et al. Accuracy of magnetically controlled capsule endoscopy, compared with conventional gastroscopy, in detection of gastric diseases. Clin Gastroenterol Hepatol 2016;14:1266-1273. e1.

31. Qian YY, Zhu SG, Hou X, et al. Preliminary study of magnetically controlled capsule gastroscopy for diagnosing superficial gastric neoplasia. Dig Liver Dis 2018;50:1041-1046.

32. Zhao AJ, Qian YY, Sun H, et al. Screening for gastric cancer with magnetically controlled capsule gastroscopy in asymptomatic individuals. Gastrointest Endosc 2018;88:466-474.e1.

33. Hu J, Wang S, Ma W, Pan D, Sun S. Magnetically controlled capsule endoscopy as the first-line examination for high-risk patients for the standard gastroscopy: a preliminary study. Scand J Gastroenterol 2019;54:934-937.

34. Xie M, Qian Y, Cheng S, Wang L, Shen R. Magnetically guided capsule endoscopy in pediatric patients with abdominal pain. Gastroenterol Res Pract 2019;2019:7172930.

35. Liao Z, Zou W, Li ZS. Clinical application of magnetically controlled capsule gastroscopy in gastric disease diagnosis: recent advances. Sci China Life Sci 2018;61:1304-1309.

36. Jiang B, Qian YY, Pan J, et al. Second-generation magnetically controlled capsule gastroscopy with improved image resolution and frame rate: a randomized controlled clinical trial (with video). Gastrointest Endosc 2020;91:1379-1387.

37. McAlindon ME, Ching HL, Yung D, Sidhu R, Koulaouzidis A. Capsule endoscopy of the small bowel. Ann Transl Med 2016;4:369.

38. Gan T, Wu JC, Rao NN, Chen T, Liu B. A feasibility trial of computer-aided diagnosis for enteric lesions in capsule endoscopy. World J Gastroenterol 2008;14:6929-6935.

39. Rondonotti E, Spada C, Adler S, et al. Small-bowel capsule endoscopy and device-assisted enteroscopy for diagnosis and treatment of small-bowel disorders: European Society of Gastrointestinal Endoscopy (ESGE) technical review. Endoscopy 2018;50:423-446.

40. McCarthy J, Minsky ML, Rochester N, Shannon CE. A proposal for the Dartmouth summer research project on artificial intelligence, August 31, 1955. AI Mag 2006;27:12-14.

41. Le Berre C, Sandborn WJ, Aridhi S, et al. Application of artificial intelligence to gastroenterology and hepatology. Gastroenterology 2020;158:76-94.e2

42. Suzuki K. Overview of deep learning in medical imaging. Radiol Phys Technol 2017;10:257-273.

43. Choi J, Shin K, Jung J, et al. Convolutional neural network technology in endoscopic imaging: artificial intelligence for endoscopy. Clin Endosc 2020;53:117-126 
44. Alaskar H, Hussain A, Al-Aseem N, Liatsis P, Al-Jumeily D. Application of convolutional neural networks for automated ulcer detection in wireless capsule endoscopy images. Sensors (Basel) 2019;19:1265.

45. Leenhardt R, Vasseur P, Li C, et al. A neural network algorithm for detection of GI angiectasia during small-bowel capsule endoscopy. Gastrointest Endosc 2019;89:189-194

46. Tsuboi A, Oka S, Aoyama K, et al. Artificial intelligence using a convolutional neural network for automatic detection of small-bowel angioectasia in capsule endoscopy images. Dig Endosc 2020;32:382-390.

47. Aoki T, Yamada A, Aoyama K, et al. Automatic detection of erosions and ulcerations in wireless capsule endoscopy images based on a deep convolutional neural network. Gastrointest Endosc 2019;89:357-363.e2.

48. Aoki T, Yamada A, Kato Y, et al. Automatic detection of blood content in capsule endoscopy images based on a deep convolutional neural network. J Gastroenterol Hepatol 2020;35:1196-1200.

49. Ding Z, Shi H, Zhang H, et al. Gastroenterologist-level identification of small-bowel diseases and normal variants by capsule endoscopy using a deep-learning model. Gastroenterology 2019;157:1044-1054.e5.

50. Rondonotti E, Koulaouzidis A, Karargyris A, et al. Utility of 3-dimen- sional image reconstruction in the diagnosis of small-bowel masses in capsule endoscopy (with video). Gastrointest Endosc 2014;80:642-651.

51. Nam SJ, Lim YJ, Nam JH, et al. 3D reconstruction of small bowel lesions using stereo camera-based capsule endoscopy. Sci Rep 2020;10:6025.

52. Gora MJ, Sauk JS, Carruth RW, et al. Tethered capsule endomicroscopy enables less invasive imaging of gastrointestinal tract microstructure. Nat Med 2013;19:238-240.

53. Cotter J, Magalhães J, de Castro FD, et al. Virtual chromoendoscopy in small bowel capsule endoscopy: new light or a cast of shadow? World J Gastrointest Endosc 2014;6:359-365.

54. Koulaouzidis A, Iakovidis DK, Yung DE, et al. Novel experimental and software methods for image reconstruction and localization in capsule endoscopy. Endosc Int Open 2018;6:E205-E210.

55. Kim HM, Kim YJ, Kim HJ, et al. A pilot study of sequential capsule endoscopy using MiroCam and PillCam SB devices with different transmission technologies. Gut Liver 2010;4:192-200.

56. Subramanian V, Ragunath K. Advanced endoscopic imaging: a review of commercially available technologies. Clin Gastroenterol Hepatol 2014;12:368-376.e1. 